\title{
Numerical treatment of free surface problems in ferrohydrodynamics
}

\author{
O Lavrova $^{1}$, G Matthies ${ }^{2}$, T Mitkova ${ }^{1}$, V Polevikov $^{3}$ and L Tobiska ${ }^{1}$ \\ ${ }^{1}$ Institute of Analysis and Numerical Mathematics, Otto-von-Guericke University Magdeburg, \\ PF 4120, D-39106 Magdeburg, Germany \\ ${ }^{2}$ Faculty of Mathematics, Ruhr University Bochum, Universitätsstraße 150, D-44780 Bochum, \\ Germany \\ ${ }^{3}$ Faculty of Applied Mathematics and Computer Science, Belarusian State University, \\ F. Skorina Avenue 4, 220050 Minsk, Belarus \\ E-mail: lavrova@mathematik.uni-magdeburg.de,gunar.matthies@ruhr-uni-bochum.de, \\ mitkova@mathematik.uni-magdeburg.de,polevikov@bsu.by and \\ tobiska@mathematik.uni-magdeburg.de
}

Received 28 April 2006, in final form 14 July 2006

Published 8 September 2006

Online at stacks.iop.org/JPhysCM/18/S2657

\begin{abstract}
The numerical treatment of free surface problems in ferrohydrodynamics is considered. Starting from the general model, special attention is paid to field-surface and flow-surface interactions. Since in some situations these feedback interactions can be partly or even fully neglected, simpler models can be derived. The application of such models to the numerical simulation of dissipative systems, rotary shaft seals, equilibrium shapes of ferrofluid drops, and pattern formation in the normal-field instability of ferrofluid layers is given. Our numerical strategy is able to recover solitary surface patterns which were discovered recently in experiments.
\end{abstract}

(Some figures in this article are in colour only in the electronic version)

\section{Introduction}

Ferrohydrodynamics (FHD) deals with the mechanics of fluid motion influenced by strong forces of magnetic polarization. In FHD, the fluid is considered as nonconducting; a principal type is a ferrofluid. Ferrofluids are colloidal suspensions of finely divided ferromagnetic or ferrimagnetic particles in a carrier liquid (water, oil, biocompatible liquids). The potential of ferrofluids for magnetic control of their properties and their flows makes them interesting for both basic research and applications; see [1]. In this paper, we will concentrate on the numerical treatment of problems in FHD where at least a part of the boundary of the domain filled with ferrofluid is unknown.

In section 2 we present a general model for describing the time-independent interactions between magnetic fields and ferrofluids with free surfaces. The mathematical model is 
governed by the coupled system of Maxwell equations and incompressible Navier-Stokes equations together with the Young-Laplace equation. This model describes field-surface as well as flow-surface interactions which are in general feedback interactions. In some cases these feedbacks are small and can be neglected partially or even fully. In this way simpler models for an efficient numerical simulation can be derived. In the following sections 3-5 we formulate typical classes of such models and give examples for their application. In section 3 we discuss a situation in which the free boundary is completely determined by the dominating magnetic forces and, thus, the hydrodynamic part is decoupled from the magnetic part. An application to dissipative systems is given. Then, in section 4, we extend this model and take into consideration feedback of the hydrodynamical forces on the free surface. This type of flow-surface interaction is important for, for example, determining the position of a ferrofluid drop in a rotary shaft seal. Even in cases where no fluid motion takes place, the magnetic field can still interact with the free surface; see section 5. Equilibrium shapes of a ferrofluid drop or a ferrofluid layer are characteristic examples of this type of field-surface interaction. We describe in detail a numerical solution strategy based on a splitting into two subproblems of solving the magnetic field equations for a given surface and calculating an equilibrium shape from given forces on the free surface. Finally, in section 6 we apply our numerical approach to find axisymmetric equilibrium surface shapes of a ferrofluid drop and equilibrium shapes of the pattern formation in normal-field instability of a ferrofluid layer. The transition between drop shapes with rounded ends and drop shapes with conical ends has been realized numerically for the first time by our methods. It is remarkable that our numerical strategy is able to recover solitary surface patterns which were discovered recently in experiments [2].

\section{Governing equations in ferrohydrodynamics}

In this section, we consider an unknown domain $\Omega_{F}$ filled with ferrofluid as a subdomain of a larger domain $\Omega \subset \mathbb{R}^{d}, d=2,3$. Let the medium in the domain $\Omega \backslash \bar{\Omega}_{F}$ be nonmagnetizable and consist of air. On the macroscopic level, the mathematical model for describing the interactions between magnetic fields and ferrofluids with free surfaces consists of the Maxwell equations, the incompressible Navier-Stokes equations, and the force balance at the unknown free surface which is given by the Young-Laplace equation; see [3]. Note that we have to consider a stress tensor for a magnetizable fluid.

The Maxwell equations for nonconducting media are given by

$$
\operatorname{curl} \mathbf{H}=\mathbf{0}, \quad \operatorname{div} \mathbf{B}=0 \quad \text { in } \Omega,
$$

with the magnetic field strength $\mathbf{H}$ and the magnetic induction $\mathbf{B}$ satisfying the relation

$$
\mathbf{B}= \begin{cases}\mu_{0}(\mathbf{H}+\mathbf{M}) & \text { in } \Omega_{F}, \\ \mu_{0} \mathbf{H} & \text { in } \Omega \backslash \Omega_{F},\end{cases}
$$

where $\mu_{0}=4 \pi \times 10^{-7} \mathrm{~V} \mathrm{~s} \mathrm{~A}^{-1} \mathrm{~m}^{-1}$ is the permeability constant and $\mathbf{M}$ denotes the magnetization. We assume that the magnetization $\mathbf{M}$ of the ferrofluid is parallel to the magnetic field $\mathbf{H}$ and that it follows the nonlinear Langevin magnetization law, i.e.,

$$
\mathbf{M}(\mathbf{H})=M_{\mathrm{S}}\left(\operatorname{coth}(\gamma H)-\frac{1}{\gamma H}\right) \frac{\mathbf{H}}{H}
$$

with the saturation magnetization $M_{\mathrm{S}}$, the Langevin parameter $\gamma=3 \chi_{0} / M_{\mathrm{S}}$, the initial susceptibility $\chi_{0}$, and $H=|\mathbf{H}|$. 
The hydrodynamical properties of the ferrofluid are described by the incompressible Navier-Stokes equations

$$
\begin{aligned}
& \rho(\mathbf{u} \cdot \nabla) \mathbf{u}-\eta \Delta \mathbf{u}+\nabla p=\mathbf{f}+\mu_{0} M \nabla H \quad \text { in } \Omega_{F}, \\
& \operatorname{div} \mathbf{u}=0 \quad \text { in } \Omega_{F},
\end{aligned}
$$

where $\rho$ denotes the fluid density, $\mathbf{u}$ the velocity, $\eta$ the dynamic viscosity, $\mathbf{f}$ a volume force, $M=|\mathbf{M}|$, and $p=p_{\mathrm{f}}+p_{\mathrm{m}}$ the (composite) pressure with the thermodynamic pressure $p_{\mathrm{f}}$ in the ferrofluid and the fluid magnetic pressure

$$
p_{\mathrm{m}}=\mu_{0} \int_{0}^{H} M\left(H^{\prime}\right) \mathrm{d} H^{\prime}
$$

We neglect the flow of air around the ferrofluid and assume a constant pressure field outside of $\bar{\Omega}_{F}$.

The system (1)-(5) is additionally coupled by the force balance at the unknown free surface $\Gamma_{F}$ between the ferrofluid and the surrounding air. Let

$\boldsymbol{\sigma}_{i j}(\mathbf{u}, p, \mathbf{H})=2 \eta \mathcal{D}(\mathbf{u})_{i j}-\left(p+\frac{\mu_{0}}{2} H^{2}\right) \delta_{i j}+B_{i} H_{j}, \quad i, j=1, \ldots d$,

denote the stress tensor $\sigma(\mathbf{u}, p, \mathbf{H})$ where the velocity deformation tensor $\mathcal{D}(\mathbf{u})$ is defined by

$$
\mathcal{D}(\mathbf{u})_{i j}=\frac{1}{2}\left(\frac{\partial u_{i}}{\partial x_{j}}+\frac{\partial u_{j}}{\partial x_{i}}\right), \quad i, j=1, \ldots d .
$$

In (6) we have $\mathbf{u}=\mathbf{0}$ and $p=p_{0}$ outside of $\bar{\Omega}_{F}$ according to the neglect of the surrounding flow field where $p_{0}$ is the atmospheric pressure. Now, the force balance on the free surface $\Gamma_{F}$ as a subset of the boundary of $\Omega_{F}$ becomes

$$
\left(\boldsymbol{\sigma}^{\text {out }}\left(\mathbf{0}, p_{0}, \mathbf{H}\right)-\boldsymbol{\sigma}^{\text {in }}(\mathbf{u}, p, \mathbf{H})\right) \mathbf{n}=\alpha \mathcal{K} \mathbf{n} \quad \text { on } \Gamma_{F} .
$$

Here, $\mathbf{n}$ is the outward unit normal vector with respect to $\Omega_{F}, \alpha>0$ the surface tension coefficient, and $\mathcal{K}$ the sum of principal curvatures.

Multiplying (7) with the normal vector $\mathbf{n}$, using the continuity of the normal component of B across the interface, we come to the Young-Laplace equation

$$
-2 \eta \mathbf{n} \cdot \mathcal{D}(\mathbf{u}) \mathbf{n}+p_{\mathrm{f}}+p_{\mathrm{m}}+\frac{\mu_{0}}{2}(\mathbf{M} \cdot \mathbf{n})^{2}=p_{0}+\alpha \mathcal{K} \quad \text { on } \Gamma_{F} .
$$

Depending on the special application we have to add further conditions at the remaining parts of the boundaries.

Note that (1), (4), (5), and (8) are a coupled system of nonlinear partial differential equations. For given $\Omega_{F}$ the equations (1)-(3) are the equations of magnetostatics in a twoor three-dimensional domain $\Omega$. For given $\Omega_{F}$ and given magnetic fields $\mathbf{H}$, (4), (5) are the incompressible Navier-Stokes equations in the two- or three-dimensional domain $\Omega_{F}$. However, in general we cannot expect the force balance (8) to be satisfied on the free surface $\Gamma_{F}$. Instead, we can consider equation (8) as a given mean curvature equation for determining the free surface and thus the shape of $\Omega_{F}$. In contrast to the case for (1)-(3) and (4), (5) we have to solve this equation for a suitable one- or two-dimensional parametrization of $\Gamma_{F}$. The existing interactions are indicated in figure 1. The shape of the free surface $\Gamma_{F}$ determines the domain $\Omega_{F}$ and influences consequently the solutions of the Maxwell and Navier-Stokes equations. Furthermore, these solutions feed back on the left-hand side of (8), i.e., there is a direct influence on the shape of $\Gamma_{F}$.

In the following sections we show how in certain cases this fully coupled system can be simplified by excluding some interactions. All numerical results presented were obtained by using the program package MooNMD; see [4]. 


\begin{tabular}{|c|c|c|c|c|}
\hline $\begin{array}{l}\text { Maxwell equations } \\
\text { magnetic field }\end{array}$ & $\Longleftrightarrow$ & $\begin{array}{l}\text { Young-Laplace equation } \\
\text { free surface }\end{array}$ & $\Longleftrightarrow$ & $\begin{array}{c}\text { Navier-Stokes equations } \\
\text { fluid flow }\end{array}$ \\
\hline
\end{tabular}

Figure 1. Coupled field-surface and flow-surface interactions.

Maxwell equations
magnetic field $\Longrightarrow \begin{gathered}\text { Young-Laplace equation } \\ \text { free surface }\end{gathered} \Longrightarrow \begin{gathered}\text { Navier-Stokes equations } \\ \text { fluid flow }\end{gathered}$

Figure 2. Dominating magnetic fields.

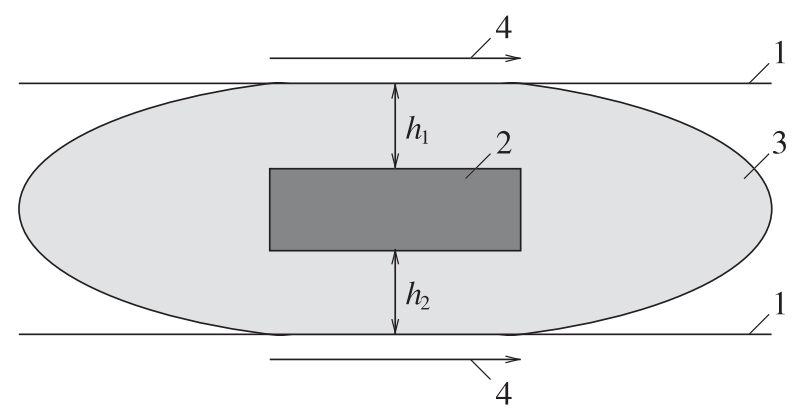

Figure 3. Geometry of a dissipative system: 1-moving plates, 2 -nonmoving magnet, 3-ferrofluid, 4-direction of the movement.

\section{Dominating magnetic fields}

In the case where magnetic forces dominate over the other forces, (8) reduces to

$$
\mu_{0} \int_{0}^{H} M\left(H^{\prime}\right) \mathrm{d} H^{\prime}+\frac{\mu_{0}}{2}(\mathbf{M} \cdot \mathbf{n})^{2}=\text { const } \quad \text { on } \Gamma_{F} .
$$

Assuming a strong magnetic field inside the fluid, such that $M \ll H$, we conclude

$$
H=\mathrm{const} \quad \text { on } \Gamma_{F},
$$

i.e., the free surface of the ferrofluid coincides with the surface of constant field intensity. Once we have calculated the magnetic field, we find the free surface shape and the hydrodynamical part can be considered as a problem in a given domain with no feedback; see figure 2 . We start with solving the Maxwell equation by neglecting the magnetization of the ferrofluid due to $M \ll H$. Therefore, a knowledge of the position of $\Omega_{F}$ is not needed. Then, having in mind a fixed given fluid volume we find from (9) the shape of the free surface $\Gamma_{F}$. Finally, the flow structure is determined by solving the Navier-Stokes equations (4), (5) in the constructed fluid domain $\Omega_{F}$.

This type of model has been applied in [5] to study numerically hydrodynamic and dissipative processes in dissipative systems consisting of a permanent magnet surrounded by a ferrofluid drop as damping medium; see figure 3. The ferrofluid drop is placed between two plates with a plane-parallel motion inducing a certain flow structure within the drop. For details on the design of such dissipative systems, we refer the reader to [6].

Assuming a rectangular shape of the permanent magnet, the magnetic field around it is analytically known, see [7], and there is no need to solve the Maxwell equations numerically. The value of the constant in (9) is determined by the given volume of the ferrofluid. Then, the field lines $H=$ const together with the two planes build the outer boundary of the fluid domain 


\begin{tabular}{|c|c|c|c|c|}
\hline $\begin{array}{l}\text { Maxwell equations } \\
\text { magnetic field }\end{array}$ & $\Longrightarrow$ & $\begin{array}{l}\text { Young-Laplace equation } \\
\text { free surface }\end{array}$ & $\Longleftrightarrow$ & $\begin{array}{c}\text { Navier-Stokes equations } \\
\text { fluid flow }\end{array}$ \\
\hline
\end{tabular}

Figure 4. Flow-surface interactions

$\Omega_{F}$. The hydrodynamic part of the model is solved numerically by a finite element method. For handling the curved boundaries, isoparametric inf-sup stable finite elements of second order have been used. More details on the discretization and the solution of the corresponding nonlinear algebraic system can be found in $[5,8]$.

We obtained numerically the values of the dissipation coefficient

$$
k=\sum_{i, j=1}^{2} \int_{\Omega_{F}}\left(\frac{\partial u_{i}}{\partial x_{j}}+\frac{\partial u_{j}}{\partial x_{i}}\right) \mathrm{d} \Omega_{F}
$$

and the flow and pressure fields for different positions of the magnet and Reynolds numbers $R e=u_{0} h \varrho / \eta$ in the range $0.1<R e<10$, where $u_{0}$ and $h$ denote the tangential velocity of the plates and the distance between the plates, respectively. The position of the magnet has been varied by looking at different ratios of its distances $h_{1}$ and $h_{2}$ to the lower and upper plates. The numerical results show that the dissipation coefficient does not depend on the Reynolds number in the above-mentioned region. The main influence on the dissipation coefficient comes from the location of the magnet inside the ferrofluid. The flow structures depend strongly on the position of the magnet. For the symmetric position $h_{1}=h_{2}$ the flow forms separate vortices above and below the magnet. However, a shift of the magnet in the direction of one of the plates causes a circular fluid motion around the magnet. For more details on the numerical results, we refer the reader to $[5,8]$.

\section{Flow-surface interactions}

In this section we consider again the case $M \ll H$ but in contrast to the case for the previous section we take into consideration hydrodynamic forces at the free surface. In such a case the solution of the Maxwell equation is determined once by neglecting the magnetization in (2). The force balance (8) can be written as

$\alpha \mathcal{K}+2 \eta \mathbf{n} \cdot \mathcal{D}(\mathbf{u}) \mathbf{n}-p_{\mathrm{f}}=\mu_{0} \int_{0}^{H} M\left(H^{\prime}\right) \mathrm{d} H^{\prime}+\frac{\mu_{0}}{2}(\mathbf{M} \cdot \mathbf{n})^{2}-p_{0} \quad$ on $\Gamma_{F}$,

where the right-hand side is a known function. Thus, we end up with a typical flow-surface interaction problem; see figure 4. As in the previous section, we can separate the problem of calculating the magnetic field. Due to capillary and normal forces at the free surface we have to consider a feedback from the flow structure on the shape of the free surface. Starting with an initial guess for the unknown domain $\Omega_{F}$, we solve the flow equations (4), (5) in $\Omega_{F}$. A new position of the free surface $\Gamma_{F}$ is found as the solution of equation (10) which is of mean curvature type. The alternating process is continued unless the change of the surface shape is smaller than a prescribed threshold. Once the magnetic field is given, the solution of (4), (5) and (10) can be found by an iterative decoupling strategy.

In some cases the model can be further simplified by neglecting capillary forces and assuming that the magnetization of the ferrofluid is in saturation, i.e., $M \approx M_{S}$. Since $M \ll H$ still holds, we also neglect the quadratic term $(\mathbf{M} \cdot \mathbf{n})^{2}$ in $(10)$ to get

$$
-2 \eta \mathbf{n} \cdot \mathcal{D}(\mathbf{u}) \mathbf{n}+\mu_{0} M_{S} H+p_{\mathrm{f}}=\mathrm{const} \quad \text { on } \Gamma_{F} .
$$




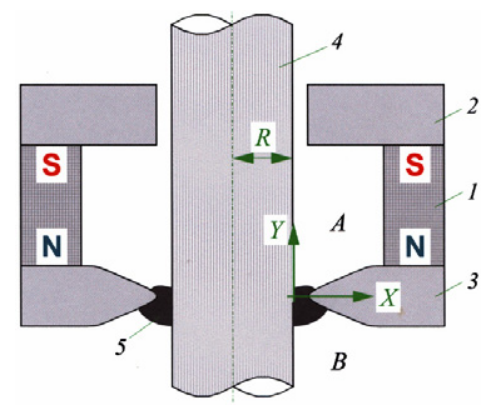

Figure 5. Schematic representation of a rotary shaft seal: 1-magnet, 2-core, 3-polar head (concentrator), 4-shaft.

We applied this type of model with flow-surface interactions to the numerical study of the ferrofluid positioning in rotary shaft seals. Rotary shaft seals are designed to admit both the rotary motion and a hermetic isolation of a high from a low pressure environment. This is realized by bringing a drop of ferrofluid into the gap between a magnet system with a hyperbolic polar head and a highly permeable rotating shaft; see figure 5. The assumption of strong magnetic fields is satisfied in practice. Moreover, due to the hyperbolic shape of the polar head, an explicit analytical expression for the magnetic field is available; see [9].

Under stationary operating conditions the ferrofluid domain $\Omega_{F} \subset \mathbb{R}^{3}$ in rotary shaft seals can be considered as rotationally symmetric. Since the minimum gap width $a$ is small compared to the radius of the rotary shaft $R$, a reduced two-dimensional model can be derived; see [8, 10]. This model consists of a transport equation for the azimuthal velocity which is coupled with the two-dimensional Navier-Stokes equations for the secondary flow and the pressure field. On the basis of the smallness for $a / R$ the convergence of an iterative decoupling strategy is proven in [8] which generalizes the idea of [9] where only one step of the iteration procedure was taken into account.

For the discretization in a domain with curved boundaries, we applied isoparametric finite elements of second order which are inf-sup stable. In [8], a detailed study of the solvability of the corresponding weak and discrete problems including a finite element error analysis has been given.

Note that in the application considered an analytical expression for the magnetic field $H$ is available. Thus, the simplified Young-Laplace equation (11) can be considered as an algebraic equation to determine the graph representations $y_{i}=y_{i}(x), i=1,2$, of the two free boundary parts from equations of the type

$$
\mu_{0} M_{S} H\left(x, y_{i}^{\text {new }}(x)\right)=G\left(x, y_{i}^{\text {old }}(x)\right)+\text { const }_{i} \quad i=1,2,
$$

where $G$ denotes a known function depending on the solution of the flow equations. For more details on the numerical study of the ferrofluid positioning in rotary shaft seals and numerical results, we refer the reader to [8-11].

\section{Field-surface interactions}

In problems of determining of equilibrium free surface shapes, the assumption of no fluid motion $\left(\mathbf{u} \equiv \mathbf{0}\right.$ in $\Omega_{F}$ ) can be made. In this case the Navier-Stokes equations (4), (5) reduce to

$$
\nabla p=-\rho g \mathbf{e}_{z}+\mu_{0} M \nabla H,
$$




\begin{tabular}{|c|c|c|c|c|}
\hline $\begin{array}{l}\text { Maxwell equations } \\
\text { magnetic field }\end{array}$ & $\Longleftrightarrow$ & $\begin{array}{l}\text { Young-Laplace equation } \\
\text { free surface }\end{array}$ & $\Longleftarrow$ & $\begin{array}{c}\text { Navier-Stokes equations } \\
\text { fluid flow }\end{array}$ \\
\hline
\end{tabular}

Figure 6. Coupled field-surface interactions.

where the volume force $\mathbf{f}$ takes into account only the gravity. Integrating the equation, we get

$$
p=-\rho g z+\mu_{0} \int_{0}^{H} M \mathrm{~d} H+p_{1}
$$

where $p_{1}$ is a constant reference pressure. Thus, the Young-Laplace equation (8) can be written in the form

$$
-\rho g z+\mu_{0} \int_{0}^{H} M \mathrm{~d} H+\frac{\mu_{0}}{2}(\mathbf{M} \cdot \mathbf{n})^{2}+C=\alpha \mathcal{K} \quad \text { on } \Gamma_{F}
$$

with the hydrostatic pressure difference $C=p_{1}-p_{0}$. The assumption of no fluid motion results in a mathematical model with field-surface interactions; see figure 6 .

For the numerical solution of the coupled system (1)-(3) and (13), we apply an iterative decoupling strategy which consists of two steps starting with an initial guess for the shape of the free surface $\Gamma_{F}$. In a first step, we solve the magnetostatic problem (1)-(3) for the given domain $\Omega_{F}$. In the second step, we find a new free surface position $\Gamma_{F}$ as the solution of the Young-Laplace equation (13) for given fields. This alternating process continues unless the change in the surface shape is smaller than a prescribed threshold. In the following we describe the numerical solution of the two subproblems in more detail.

\subsection{Numerical treatment of the magnetostatic problem}

Since curl $\mathbf{H}=\mathbf{0}$, the magnetic field can be expressed in terms of a scalar magnetic potential $\varphi$ such that $\mathbf{H}=-\nabla \varphi$. It follows from (1)-(3) that

$$
\begin{aligned}
& -\operatorname{div}(\mu(\xi,|\nabla \varphi|) \nabla \varphi)=0 \\
& \mu(\xi,|\nabla \varphi|)= \begin{cases}1+\frac{M(|\nabla \varphi|)}{|\nabla \varphi|} & \text { in } \Omega, \\
1 & \xi \in \Omega_{F},\end{cases}
\end{aligned}
$$

The interface conditions between two media with different magnetic properties are given by

$$
\varphi^{\text {in }}=\varphi^{\text {out }}, \quad\left(1+\frac{M\left(\left|\nabla \varphi^{\text {in }}\right|\right)}{\left|\nabla \varphi^{\text {in }}\right|}\right) \frac{\partial \varphi^{\text {in }}}{\partial n}=\frac{\partial \varphi^{\text {out }}}{\partial n} \quad \text { on } \Gamma_{F} ;
$$

see [3]. Here, the superscripts in and out denote the variables in the fluid and the air domains, respectively. Let us assume that $\Omega \backslash \Omega_{F}$ is an unbounded domain and far from the fluid the magnetic field is uniform with the intensity $H_{0}$ :

$$
\lim _{|\xi| \rightarrow \infty}\left(\varphi-H_{0} z\right)=0 .
$$

If the fluid domain $\Omega_{F}$ is bounded than a strategy of coupling boundary and finite element methods (BEM-FEM approach) can be applied to solve the magnetostatic problem (14)-(16). A boundary element method is used in the unbounded air domain $\Omega \backslash \Omega_{F}$ to impose exactly the boundary condition (16) at infinity while a finite element method resolves the nonlinearity of the magnetostatic equations in $\Omega_{F}$ accurately. The BEM-FEM approach has been used in [12-14] for the problem of determining axisymmetric equilibrium surface shapes of a ferrofluid drop under the influence of a uniform magnetic field and for the problem on equilibrium shapes of a ferrofluid layer in a plane capillary subjected to a uniform magnetic field; see [15]. 
If the fluid domain $\Omega_{F}$ is unbounded and the nonlinear magnetization law (3) is essential, we cannot use the BEM in the unbounded domain. Therefore, we construct a cut bounded computational domain $\widetilde{\Omega} \subset \Omega$ and apply the finite element method (FEM) to solve the magnetostatic problem (14)-(16) in $\widetilde{\Omega}$. Hence, we have to define suitable boundary conditions for the magnetostatic potential on $\partial \widetilde{\Omega}$. Let us explain this in the case of a magnetic fluid layer $\Omega_{F}$ which occupies initially the region $z \leqslant 0$ and a uniform magnetic field $\mathbf{H}=\left(0,0,-H_{0}\right)$ at infinity. We cut $\Omega=\mathbb{R}^{3}$ with artificial boundaries $z=z_{\text {bottom }}<0$ and $z=z_{\text {top }}>0$. The distances of these boundaries from the free surface should be large enough to ensure that the magnetic field is uniform there, i.e.

$$
\varphi=H_{F} z_{\text {bottom }} \quad \text { for } z=z_{\text {bottom }}, \quad \varphi=H_{0} z_{\text {top }} \quad \text { for } z=z_{\text {top }} .
$$

Since the magnetic field is perturbed by the surface only in the neighbourhood of the interface, we get from the second condition in (15) applied to the flat surface $z=0$

$$
\left(1+\frac{M\left(H_{F}\right)}{H_{F}}\right) H_{F}=H_{0} .
$$

Solving this nonlinear equation by the Newton method, we obtain the value of $H_{F}$ in (17). The projection of the computational domain onto the $x-y$ plane has to be also restricted to a bounded domain $\Omega^{\prime} \in \mathbb{R}^{2}$ such that $\tilde{\Omega}=\Omega^{\prime} \times\left[z_{\text {bottom }}, z_{\text {top }}\right]$. In the case of a pattern developed in the Rosensweig instability, one could choose a set of hexagons or squares as $\Omega^{\prime}$ and a symmetry condition at the vertical boundaries of $\tilde{\Omega}$ :

$$
\frac{\partial \varphi}{\partial n}=0 \quad \text { on } \partial \Omega^{\prime} \times\left(z_{\text {bottom }}, z_{\text {top }}\right)
$$

A FEM approach for solving (14) under the conditions (15), (17), (18) has been applied to study the Rosensweig instability for example in [10, 16-18].

\subsection{Numerical treatment of the free surface}

Let us assume that the free surface $\Gamma_{F}$ is of graph type:

$$
\Gamma_{F}=\left\{(x, y, z) \mid z=Z(x, y),(x, y) \in \Omega^{\prime}\right\} .
$$

Substituting the curvature, expressed in terms of the function $Z(x, y)$, into (13) we get

$-\alpha \operatorname{div} \frac{\nabla Z}{\sqrt{1+|\nabla Z|^{2}}}+\rho g Z=\mu_{0} \int_{0}^{H} M \mathrm{~d} H+\frac{\mu_{0}}{2}(\mathbf{M} \cdot \mathbf{n})^{2}+C \quad$ in $\Omega^{\prime}$.

Equation (19) together with the symmetry boundary condition

$$
\frac{\partial Z}{\partial n}=0 \quad \text { on } \partial \Omega^{\prime}
$$

is solved in respect to the function $Z(x, y)$ for the given magnetic field by the finite element method. For the details on the discretization methods see $[10,17,18]$.

Many physical problems can be reasonably described in a two-dimensional setting. Here, we focus on two classes of such problems, namely, axisymmetric and plane ones. Under the assumption of axial symmetry, three-dimensional problems can be reformulated as twodimensional ones in the meridian plane. The plane problems are described using mathematical models in a vertical cross section of a three-dimensional domain.

Let $\Omega \subset \mathbb{R}^{2}$ be the meridian plane or the vertical cross section. Then, $\Gamma_{F}$ is a curve which can be represented in a parametric form

$$
\Gamma_{F}=\{(x, y) \mid x=X(s), y=Y(s), s \in[0, \ell]\} .
$$


Let us take the arc length of the free surface $\Gamma_{F}$ as a parameter $s$. For the parametrization with respect to the arc length, we have

$$
X^{\prime 2}+Y^{\prime 2}=1 \quad s \in[0, \ell] .
$$

The curvature of a curve is expressed as $\mathcal{K}=-Y^{\prime \prime} / X^{\prime}$, where $s$ goes clockwise along $\Gamma_{F}$. For axisymmetric problems, the curvature is a sum of a meridional sectional curvature $-Y^{\prime \prime} / X^{\prime}$ and a latitudinal sectional curvature $-Y^{\prime} / X$. The Young-Laplace equation results in

$-\alpha\left(\frac{Y^{\prime \prime}}{X^{\prime}}+k \frac{Y^{\prime}}{X}\right)+\rho g Y=\mu_{0} \int_{0}^{H} M \mathrm{~d} H+\frac{\mu_{0}}{2}(\mathbf{M} \cdot \mathbf{n})^{2}+C, \quad s \in[0, \ell]$

where $k=0$ for the plane problem and $k=1$ for the axisymmetric problem.

A numerical approach for the numerical solution of (20), (21) with the a priori unknown value of $\ell$ was elaborated in [19]. It has been successfully applied in [12-14] to the problem on axisymmetric equilibrium surface shapes of a ferrofluid drop under the influence of a uniform magnetic field, in [15] to the problem on equilibrium shapes of a ferrofluid layer in a plane capillary subjected to a uniform magnetic field, and in $[10,16]$ to the numerical study of the Rosensweig instability.

\section{Some results on equilibrium shapes}

One of the classical problems in ferrohydrostatics is finding an equilibrium of a free ferrofluid drop in an external magnetic field; see [3]. The freely suspended ferrofluid drop, initially held spherical by surface tension, elongates in the direction of the uniform applied magnetic field and takes stable equilibrium shapes.

At sufficiently large values of the magnetic susceptibility $\chi_{0}$, the drop deformation can exhibit hysteresis. Modestly deformed prolate drops jump to much more elongated shapes when the magnetic field increases above a certain threshold. These large elongations persist even when the magnetic field decreases below the critical value. For decreasing magnetic field, a jump from the upper branch of solutions with large elongated shapes to the lower branch of solutions appears at a second threshold. A limited range of the magnetic field strength exists (hysteretic regime) where two equilibrium configurations of a drop can be observed: one occurs upon increasing the strength of the magnetic field, the another upon its decrease. Experimental measurements of hysteresis in the deformation of agglomerate ferrofluid drops $(2-20 \mu \mathrm{m})$ were reported for the first time in [20]. According to the theoretical study in [20] which is based on the energy minimization under the assumption of spheroidal drop shapes, the drop deformation displays hysteresis as a function of the applied field for $\chi_{0}>20$. We observed numerically that the dependence of the drop elongation upon the field goes from a monotone curve for $\chi_{0}<21$ to S-shaped curves for $\chi_{0} \geqslant 21$; see figure 7(a). Figure 7(a) shows that increase of the magnetic field up to the magnetic Bond number $B_{\mathrm{m}}=5.723$ results in a continuous drop elongation. A slight change from $B_{\mathrm{m}}=5.723$ to 5.725 causes a jump in the drop elongation accompanied by a transition from a shape with rounded ends to a shape close to conical; see figure 7(b). Such a transition was for the first time realized numerically.

According to the results of numerical simulations, we observe that in the vicinity of the symmetry axis the drop shape becomes acute for all solutions lying on the upper branch; see figure 7(b). Shapes close to conical appear after the first turning point for increasing magnetic field and preserve their acute configuration to the second turning point for decreasing field. We found that the difference between theory and numerics lies within $1 \%$ for the lower branch of the solution, but shows more than $15 \%$ for the upper branch of the hysteretic curve. Numerically resolved equilibrium shapes with pointed ends result in the strong deviation from spheroidal shapes of the theory. 


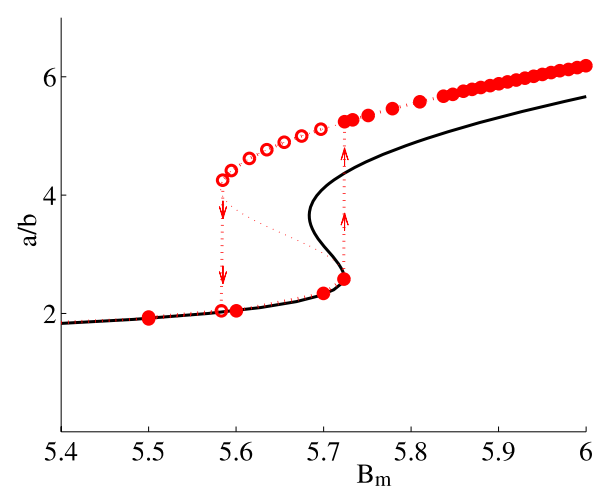

(a)

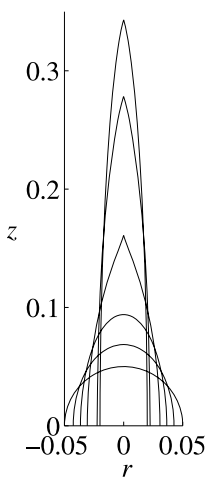

(b)

Figure 7. (a) The dependence of the drop elongation upon the magnetic Bond number $B_{\mathrm{m}}=$ $\mu_{0} V^{1 / 3} \chi_{0} H_{0} 2 /(2 \alpha)$ for $\chi_{0}=21$. Here, $V$ denotes the drop volume, $a$ and $b$ are drop lengths along $z$ and $r$ axes, respectively. The solid line corresponds to the spheroidal drop approximation in [20], full (empty) markers present numerical results for increasing (decreasing) field. (b) Equilibrium shapes for $B_{\mathrm{m}}=\{0,5,5.723,5.725,10,15\}, \chi_{0}=21$.

One of the most spectacular phenomena shown by ferrofluids is the Rosensweig or normalfield instability; see [3]. It occurs when a ferrofluid vessel is brought into a magnetic field which is perpendicular to the flat and horizontal ferrofluid-air interface. For small magnetic field strength the interface remains flat while a spontaneous surface deformation into a hexagonal patterns takes place if the field strength exceeds a certain threshold $H_{\mathrm{c}}$. Furthermore, the height of the peaks in the pattern increases with increasing magnetic field. If the strength of the applied magnetic field is decreased then the peak height reduces. If the magnetic field strength is below its critical value $H_{\mathrm{c}}$, the surface deformation persists until the field strength falls below a second threshold. The surface deformation shows a hysteretic behaviour with changing field.

Using a Langevin fit through an experimentally obtained magnetization curve, we succeeded in obtaining numerical results which agree with the experimental data very well. For comparing both the experimental and numerical results, we introduce the dimensionless parameter $\varepsilon=\left(H^{2}-H_{\mathrm{c}}^{2}\right) / H_{\mathrm{c}}^{2}$, where $H_{\mathrm{c}}$ is the critical magnetic field strength for the onset of the instability. Note that the critical magnetic field strength was chosen to be the observed value instead of the theoretical one which is predicted by a linear stability analysis; see e.g. [3]. The values obtained for $H_{\mathrm{c}}$ in the experiment and the numerical simulation differ only slightly. A comparison of the peak height as a function of $\varepsilon$ is shown in figure 8(a). An almost perfect match between the experimental data and the numerical results can be observed. For a detailed study, we refer the reader to [21].

The peak shape obtained is presented in figure 8(b). Here, we see again that the numerical simulation and the experimental data fit perfectly even for rather big values of the bifurcation parameter $\varepsilon$. For more details, see [21].

A solitary surface pattern (soliton) was recently discovered experimentally in the bistability interval of the Rosensweig instability; see [2]. A single stationary peak was generated by a local field perturbation in the hysteretic regime of the Rosensweig instability and observed to be stable after turning off the additionally applied local field. On the basis of experimental observations we can interpret a solitary surface configuration as an additional stable state in the hysteretic regime beside the flat surface and the fully developed pattern. This assumption allows us to apply the numerical approach of section 5 to resolve a solitary peak as an equilibrium state of the Rosensweig instability. 


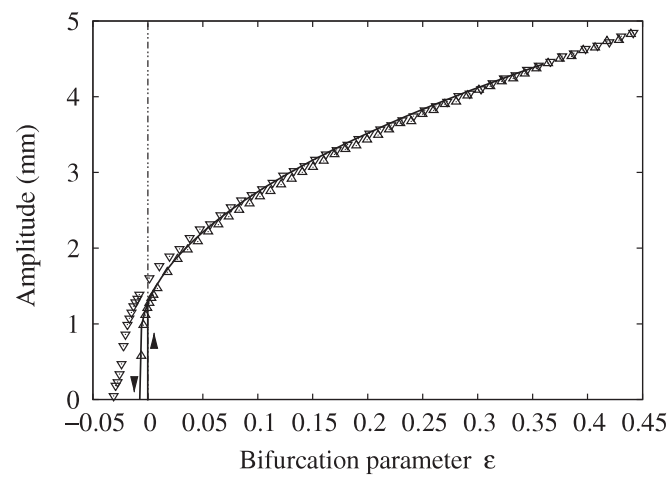

(a)

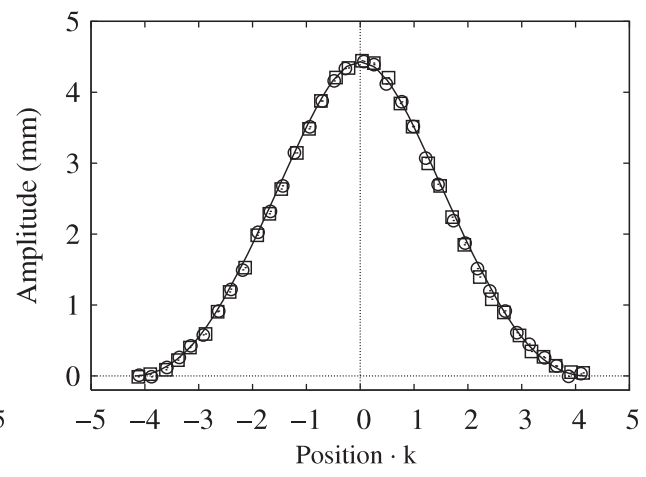

(b)

Figure 8. (a) Pattern amplitude as a function of the bifurcation parameter $\varepsilon=\left(H^{2}-H_{\mathrm{c}}^{2}\right) / H_{\mathrm{c}}^{2}$. $H_{\mathrm{c}}$ denotes the field intensity at which the jump occurs when increasing the field. The solid line shows numerical results, whereas the experimental data are represented by the upward (downward) triangles for increasing (decreasing) magnetic field. (b) Comparison of experimentally and numerically obtained peak profiles at $\varepsilon=0.35$, normalized to the wavelength. The wavelength for the simulations is fixed by the theoretically predicted wavelength of the pattern $\lambda=2 \pi \sqrt{\alpha /(\rho g)}$; see e.g. [3].

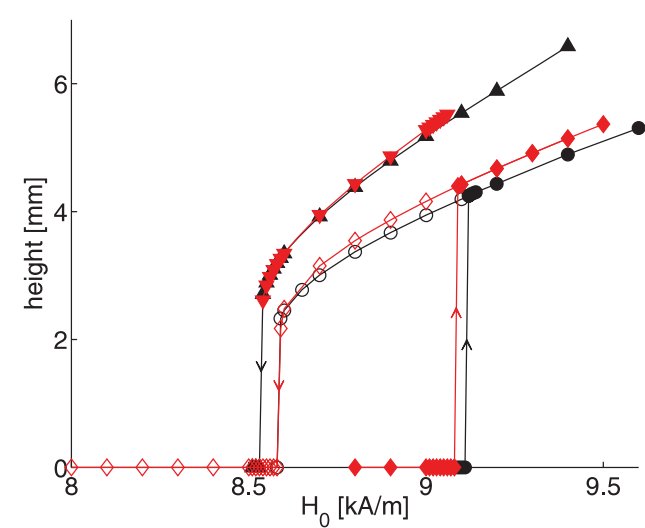

(a)

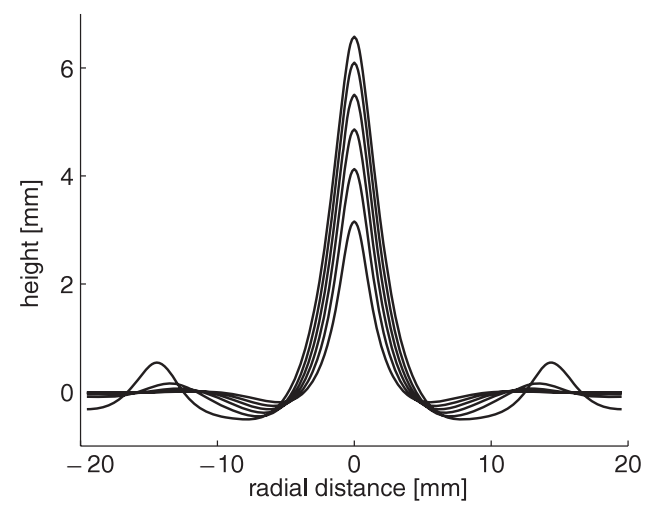

(b)

Figure 9. (a) The dependence of the peak height upon the applied field. The full (empty) circles show the height of the axisymmetric pattern approximation for the increasing (decreasing) field. The full (empty) diamonds present the height of the fully three-dimensional hexagonal pattern for the increasing (decreasing) field. The upward (downward) triangles give the height of the axisymmetric (three-dimensional) soliton. (b) Soliton shapes for $H_{0}=\{8.6,8.8,9,9.2,9.4,9.6\} \mathrm{kA} \mathrm{m}^{-1}$; axisymmetric model. The magnetic fluid EMG 901 with the parameters $\chi_{0}=2.2, \rho=$ $1406 \mathrm{~kg} \mathrm{~m}^{-3}, \alpha=0.025 \mathrm{~kg} \mathrm{~s}^{-2}, M_{\mathrm{S}}=48 \mathrm{kA} \mathrm{m}^{-1}$ is used.

Primarily, we perform numerical simulations of the Rosensweig instability to resolve a hysteretic regime for a magnetic fluid EMG901. Both axisymmetric equilibrium shapes and fully three-dimensional shapes are calculated. In figure 9(a) the height of the numerically resolved peaks over the magnetic field strength is shown. The hysteresis effect is observed within a large bistability interval

$$
8.59 \pm 0.01 \mathrm{kA} \mathrm{m}^{-1} \leqslant H_{0} \leqslant 9.12 \pm 0.01 \mathrm{kA} \mathrm{m}^{-1}
$$


To resolve single peak configurations on the free surface, we set the applied field $H_{0}$ to $9 \mathrm{kA} \mathrm{m}^{-1}$. We specify an enlarged computational domain such that it covers a multiple of the pattern wavelength and prescribe a symmetry condition at the boundary. The initial local field perturbation applied in the experiment for a soliton generation is replaced in numerical simulations by an initial surface deformation. After getting a soliton as an equilibrium surface shape, we resolve a whole family of solitary configurations by increasing and decreasing the strength of the applied field with a step size of $0.01 \mathrm{kA} \mathrm{m}^{-1}$; see figure 9(a).

The numerical results shown in figure 9(a) agree qualitatively with experimental results; see [2, figure 3]. At the same field strength, a soliton has a higher amplitude than a peak in the pattern. An increase of the field strength results in an increasing height of a soliton, while for the decreasing field the peak amplitude decreases. Figure 9(b) displays soliton shapes for different values of the applied magnetic field. We see that a stronger intensity of the magnetic field results in a higher peak amplitude and a deeper circular hollow around the soliton. Soliton configurations are experimentally observed within the bistability interval with a sudden transition to the fully developed Rosensweig pattern for the field $H_{0} \approx H_{\mathrm{c}}+0.02 \mathrm{kA} \mathrm{m}^{-1}$, slightly stronger than the critical one. Results of numerical calculations predict the existence of soliton configurations in a wider range of field intensities; see figure 9(a). This numerical finding should be further studied.

\section{Conclusions}

Starting from the fundamental equations of the mathematical model, our presented numerical methods are able to give insight into the behaviour of ferrofluids in the presence of magnetic fields. Depending on the problem considered, the feedback interactions between the Maxwell equations, the Navier-Stokes equations, and the Young-Laplace equation can be neglected partially or even fully.

The paper suggests a free surface problem classification in ferrohydrodynamics based on the nature of field-surface and flow-surface interactions. A reliable numerical treatment for every class of problems is proposed and verified on a variety of applications.

In the simulation of equilibrium drop shapes, the transition between shapes with rounded ends and shapes with conical ends was obtained for the first time numerically.

The solitary surface patterns which were recently observed experimentally have been recovered by our numerical methods.

\section{Acknowledgments}

The authors would like to thank Viktor Bashtovoi and Reinhard Richter for the fruitful cooperation. Financial support by the German Research Foundation (DFG) under grants within the high priority research programme SPP 1104 as well as by the German Academic Exchange Service (DAAD) is gratefully acknowledged.

\section{References}

[1] Odenbach S (ed) 2002 Ferrofluids (Springer Lecture Notes in Physics vol 594) (Berlin: Springer)

[2] Richter R and Barashenkov I V 2005 Phys. Rev. Lett. 94184503

[3] Rosensweig R 1997 Ferrohydrodynamics (New York: Dover)

[4] John V and Matthies G 2004 Comput. Vis. Sci. 6 163-70

[5] Bashtovoi V, Lavrova O, Mitkova T, Polevikov V and Tobiska L 2005 J. Magn. Magn. Mater. 289 207-10 
[6] Bashtovoi V, Kabachnikov D, Kolobov A, Samoylov A and Vikoulenkov A 2002 J. Magn. Magn. Mater $252312-4$

[7] Pshenichnikov A 1993 Magnetohydrodynamics 29 33-6

[8] Mitkova T 2004 Lösbarkeit und FE-Approximation eines mathematischen Modells für die Strömung in Magnetfluiddichtungen PhD Thesis University Magdeburg

[9] Polevikov V and Tobiska L 2001 Fluid Dyn. 36 890-8

[10] Lavrova O, Matthies G, Mitkova T, Polevikov V and Tobiska L 2003 Challenges in Scientific Computing-CISC 2002 ed E Bänsch (Berlin: Springer) pp 161-83

[11] Mitkova T and Tobiska L 2004 Large-Scale Scientific Computing (Springer Lecture Note in Computer Science vol 2907) ed I Lirkov et al (Berlin: Springer) pp 396-403

[12] Lavrova O 2006 Numerical methods for axisymmetric equilibrium magnetic-fluid shapes $P h D$ Thesis University Magdeburg

[13] Lavrova O, Matthies G, Polevikov V and Tobiska L 2004 Proc. Appl. Math. Mech. 4 704-5

[14] Lavrova O, Polevikov V and Tobiska L 2005 Proc. Appl. Math. Mech. 5 837-8

[15] Polevikov V and Tobiska L 2005 J. Magn. Magn. Mater. 289 379-81

[16] Bashtovoi V, Lavrova O, Polevikov V and Tobiska L 2002 J. Magn. Magn. Mater. 252 299-301

[17] Matthies G 2002 Finite element methods for free boundary value problems with capillary surfaces PhD Thesis University Magdeburg

[18] Matthies G and Tobiska L 2005 J. Magn. Magn. Mater. 289 346-9

[19] Polevikov V 2004 Comput. Methods Appl. Math. 4 66-93

[20] Bacri J C and Salin D 1982 Phys. Lett. 43 649-54

[21] Gollwitzer C, Matthies G, Richter R, Rehberg R and Tobiska L 2006 The surface topography of a magnetic fluid a quantitative comparison between experiment and numerical simulation J. Fluid Mech. at press 